\title{
Failure Mechanisms of a Gold Microelectrode in Bioelectronics Applications
}

\author{
Jonghun Kim and Sang-Hee Yoon \\ Micro/Nano Soft Biomechanics Laboratory, Department of Mechanical Engineering, Inha University, 100 Inha-ro, Nam-gu, \\ Incheon 402-751, Republic of Korea \\ Correspondence should be addressed to Sang-Hee Yoon; shyoon@inha.ac.kr
}

Received 5 October 2014; Accepted 16 November 2014

Academic Editor: Jong-Yeop Kim

Copyright (C) 2015 J. Kim and S.-H. Yoon. This is an open access article distributed under the Creative Commons Attribution License, which permits unrestricted use, distribution, and reproduction in any medium, provided the original work is properly cited.

\begin{abstract}
The generation, growth, and collapse of tiny bubbles are inevitable for a microelectrode working in aqueous environment, thus resulting in physical damages on the microelectrode. The failure mechanisms of a microelectrode induced by tiny bubble collapsing are investigated by generating tiny hydrogen bubbles on a gold microelectrode through deionized water electrolysis. The surface of the microelectrode is modified with a thiol-functionalized arginine-glycine-aspartic acid peptide to generate perfectly spherical bubbles in proximity of the surface. The failure of an Au microelectrode is governed by two damage mechanisms, depending on the thickness of the microelectrode: a water-hammer pressure due to the violent collapse of a single large bubble, formed through merging of small bubbles, for ultrathin Au microelectrodes of 40-60 nm in thickness, and an energy accumulation resulting from the repetitive collapse of tiny bubbles for thick Au microelectrodes of 100-120 nm.
\end{abstract}

\section{Introduction}

Bioelectronics creates innovative devices or processes for the diagnosis, treatment, prognosis, and prevention of diseases through the application of electrical engineering principles to biology or medicine. For bioelectronics applications where a microelectrode is commonly used in aqueous environment to electrically stimulate biological tissues, the generation, growth, and collapse of tiny bubbles by electrolysis of water (or other solutions) are an unavoidable consequence. An enormous concentration of energy resulting from bubble collapse is known to be responsible for the occurrence of microscopic damages on a microelectrode [1] and the deleterious effects on a biological system (e.g., hemolysis, renal injury, etc.) $[2,3]$. This phenomenon not only is restricted to bioelectronics, but also frequently involves a variety of macroscale engineering applications (e.g., pumps, turbines, propellers, bearings, etc.) $[4,5]$ to nano-/microscale ones (e.g., nano-/microelectromechanical systems (N/MEMS), semiconductors, etc.) $[6,7]$.

Consequently, the physics in a tiny bubble (especially, bubble collapsing) has been studied quite intensively for many years to understand the bubble dynamics and to investigate the damage mechanism of solid substrates caused by bubble cavitation $[1,8,9]$. Although the previous approaches have been successful in theoretically understanding bubble collapse, they fail to answer a number of basic questions regarding the fundamental failure mechanisms involved. The reason is that this problem is related to unsteady two-phase flow combined with the reaction of the specific material of which a substrate is made.

Here, we generate tiny hydrogen bubbles, through deionized (DI) water electrolysis, on a gold microelectrode whose surface is modified with a thiol-functionalized arginineglycine-aspartic acid peptide (RGD peptide) for heterogeneous bubble nucleation in proximity of the surface. This leads to the quantitative characterization of the failure mechanisms of an Au microelectrode due to tiny bubble collapsing and establishes an empirical rule for evaluating the possibility of cavitation damage on an Au microelectrode. Furthermore, we provide experimental evidence both for supporting our findings and for visualizing physical damages on an $\mathrm{Au}$ microelectrode in nano-/microscale. 


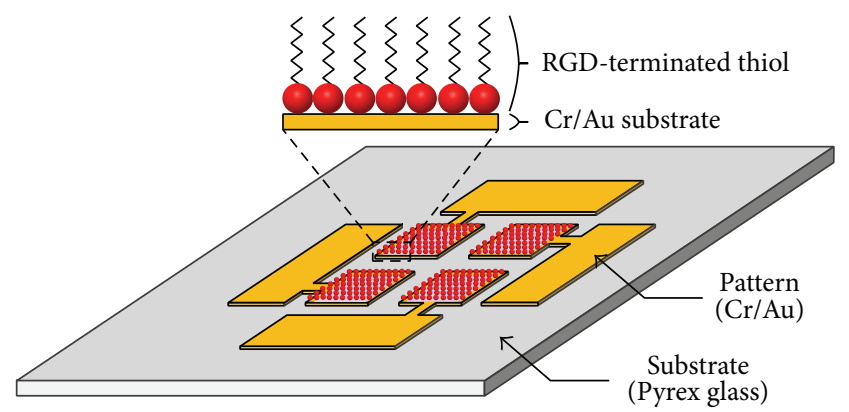

FIGURE 1: An experimental sample for characterizing the failure mechanisms of a gold electrode induced by tiny bubble collapsing. The sample is composed of four identical $\mathrm{Au}$ microelectrodes patterned on a Pyrex glass substrate, the surface of each of which is modified with a thiol-functionalized RGD peptide for heterogeneous bubble nucleation in proximity of the surface.

\section{Materials and Methods}

Experimental samples consisted of identical Au microelectrodes patterned on a Pyrex substrate, which made it possible to collect a lot of experimental results from a single sample, as shown in Figure 1. The Au microelectrodes were modified with a thiol-functionalized RGD peptide to make the microelectrode surface hydrophilic and moderate (in roughness). This was intended to achieve bubble generation in proximity of the surface. The RGD peptide was tethered to the Au microelectrodes via thiol compound by a spontaneous chemisorption of R-S-H $+\mathrm{Au} \rightarrow \mathrm{R}-\mathrm{S}-\mathrm{Au}+(1 / 2) \mathrm{H}_{2}$, where $\mathrm{R}$ is a substituent [10].

The preparation of the experimental samples began with a Pyrex glass wafer. After piranha cleaning, a double-layer resist stack composed of $1 \mu \mathrm{m}$ thick LOR resist (LOR $10 \mathrm{~A}$, MicroChem Corp.) and $2 \mu \mathrm{m}$ thick positive photoresist (S1818, Rohm and Haas Corp.) was spin-coated and patterned, followed by the deposition of $5 \mathrm{~nm}$ thick Cr adhesion layer and 40 to $120 \mathrm{~nm}$ thick Au layer through e-beam evaporation. Next, the double-layer resist stack was lifted off to fabricate the experimental samples, each of which consisted of four microelectrodes of $500 \times 500 \mu \mathrm{m}^{2}$ square [11]. The surface of the Au microelectrodes was treated with a thiolfunctionalized RGD peptide whose solution was synthesized by chemically combining cyclo (Arg-Gly-Asp-D-PheLys) [c(RGDfK), $\mathrm{C}_{27} \mathrm{H}_{41} \mathrm{~N}_{9} \mathrm{O}_{7}$, Peptides International, Inc.] with dithiobis(succinimidyl undecanoate) $\left(\mathrm{C}_{30} \mathrm{H}_{48} \mathrm{~N}_{2} \mathrm{O}_{8} \mathrm{~S}_{2}\right.$, Dojindo Molecular Technologies, Inc.). For the surface modification, the experimental samples were incubated with the solution for 1 hour at room temperature to promote a spontaneous chemisorption between thiol and gold [12].

The failure process in Au microelectrodes was observed with an experimental apparatus composed of a glass petri dish containing the experimental sample and DI water [or 1X Dulbecco's phosphate buffered saline solution (DPBS, D8537, Sigma-Aldrich Co. LLC.)], an upright microscope (BX$51 \mathrm{M}$, Olympus Corporation) with a color charge-coupled device (CCD) camera (DP25, Olympus Corporation), and a platinum tip coupled with a DC power supply (PWS2000,

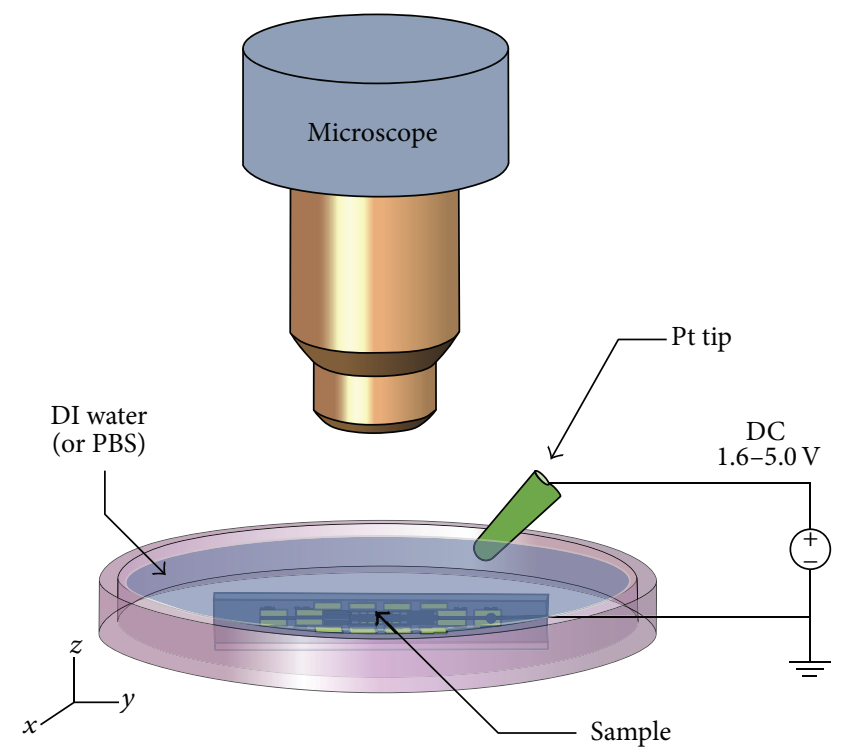

FIGURE 2: Experimental apparatus for observing the failure of the $\mathrm{Au}$ microelectrode due to tiny bubble collapsing. Tiny hydrogen bubbles are generated by electrolyzing DI water or 1X DPBS.

Tektronix, Inc.), as shown in Figure 2. We applied negative DC voltage of $-7.0 \mathrm{~V}$ to $-1.6 \mathrm{~V}$ to the experimental samples to generate hydrogen bubbles thereon, the nature of which was controlled by adjusting the level of DC voltage. The changes (especially failure) in the surface of the experimental samples induced by tiny bubble collapse were observed and recorded with the optical microscope.

The surface profile of the physical damages on Au microelectrodes was measured with an atomic force microscopy (AFM) system (Park XE7, Park Systems Corporate). The topography was made in a constant height mode and therefore the deflection of an AFM tip [ACL probe, $58.0 \mathrm{~N} / \mathrm{m}$ (nominal spring constant), Applied NanoStructures, Inc.] under scanning reflected the topography of the Au microelectrode. The real spring constant of the AFM tip was determined as $67.2 \pm 0.2 \mathrm{~N} / \mathrm{m}$ after calibration, which was used in the AFM measurement.

\section{Results and Discussion}

3.1. Tiny Hydrogen Bubble Generation on Gold Microelectrode. An electrolysis of DI water produced tiny hydrogen bubbles with an average radius of $19.1-38.9 \mu \mathrm{m}$ on the $\mathrm{Au}$ microelectrode surface modified with a thiol-functionalized RGD by electrolyzing when applied voltage was adjusted at a range of $1.6 \mathrm{~V}$ to $7.0 \mathrm{~V}$. As a first step, the mean (including minimum and maximum) radius of the tiny bubbles was measured as a function of applied voltage to characterize the sensitivity of tiny bubble generation to variation in the electrical input, as shown in Figure 3(a). It was observed that the threshold voltage (i.e., the minimum voltage required to generate noticeable tiny bubbles) was about $1.5-1.6 \mathrm{~V}$, a little higher than the decomposition potential of water (i.e., $1.2 \mathrm{~V}$ ). Next, while the bubble size increased as the voltage increased 


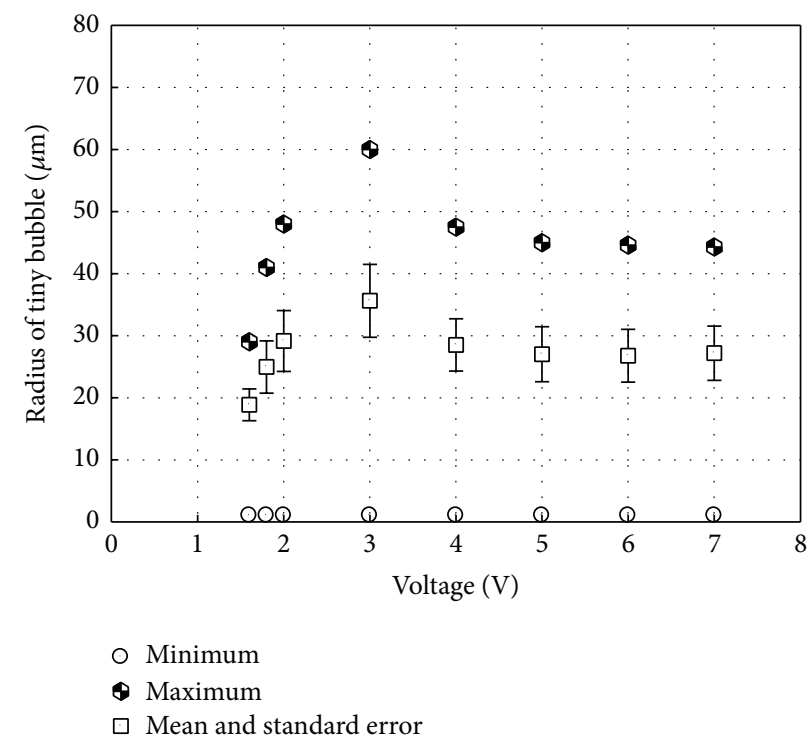

(a)

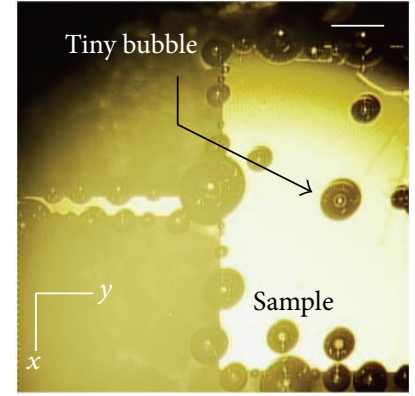

(b)

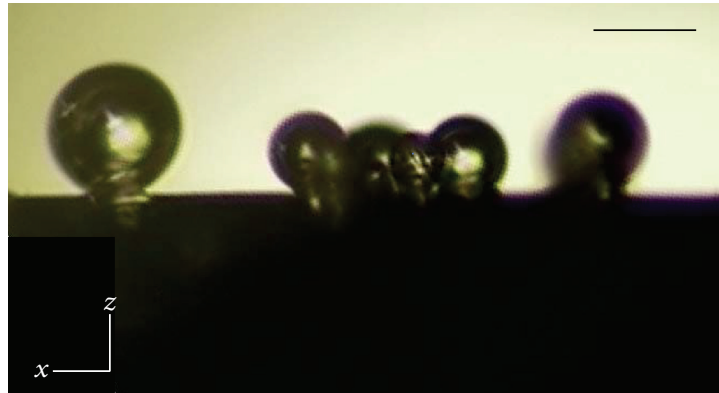

(c)

FIGURE 3: Tiny hydrogen bubble generation on the Au microelectrode through electrolyzing an electrolyte (i.e., DI water or 1X DPBS). (a) The size of the electrolytically generated bubble as a function of applied voltage. An increase in the voltage to $3.0 \mathrm{~V}$ leads to an increase in the bubble size and further increase in the voltage (i.e., higher than $3.0 \mathrm{~V}$ ) results in decrease and saturation in the bubble size. No change in the minimum size of all bubbles indicates no change in the size of bubble nucleation sites. ((b)-(c)) Top (b) and side (c) views of the tiny hydrogen bubbles on the surface of the Au microelectrode. The microscopic images show the tiny hydrogen bubbles are formed into a perfect sphere in proximity of the surface. Scale bars of (b) and (c) are $50 \mu \mathrm{m}$.

from $1.6 \mathrm{~V}$ to about $3.0 \mathrm{~V}$, it started to decrease and saturate when the voltage was higher than $3.0 \mathrm{~V}$. This is in contrast to porous plate bubbler generation where an increase in gas generation is closely associated with an increase in bubble size [13]. This shows that an increase in the voltage leads to an increase in both gas (i.e., hydrogen) production and the degree of violence in the merging process of small bubbles. An increase in the voltage from $1.6 \mathrm{~V}$ to $3.0 \mathrm{~V}$ at which there is no or mild merging process of small bubbles into big one leads to an increase in gas production, therefore resulting in an increase in bubble size. A further increase in the voltage (i.e., higher than $3.0 \mathrm{~V}$ ) accompanying the violent merging process of small bubbles, however, makes the bubble size decrease and then saturate due to the instability in bubble caused during the violent merging process. Noticeably, the fact that the radius of the bubbles is less than $50 \mu \mathrm{m}$ shows that an electrolysis of DI water is suited to be used as a source of tiny bubble generation in characterizing the failure mechanisms of microelectrodes (or micropatterns), compared to conventional porous plate bubblers that produce bubbles with a radius of much bigger than $100 \mu \mathrm{m}$ [13].

The electrolytically generated bubbles on the Au microelectrode had a perfectly spherical form in proximity of the surface, as shown in Figures 3(b) and 3(c). The reason is that the surface of the microelectrode is modified with a thiol-functionalized RGD. This surface treatment is known to make Au surface hydrophilic and moderate in roughness [12], which are favorable conditions for bubble generation in proximity of the surface. Meanwhile, for each tiny bubble generated, heterogeneous nucleation (i.e., nucleation formation at an interface between any two phases of gas, liquid, or solid) was seen. As might be expected, this is because the energy barrier for homogeneous nucleation is higher than that for heterogeneous nucleation. The top and side views of the bubbles provide us with information about a standoff parameter, $\gamma$. As a core nondimensional parameter in 

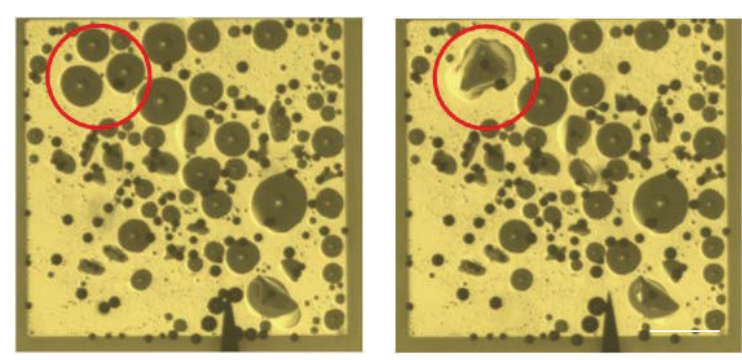

(a)
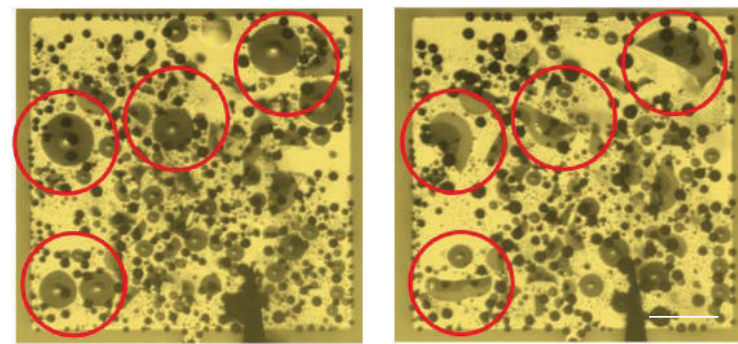

(c)
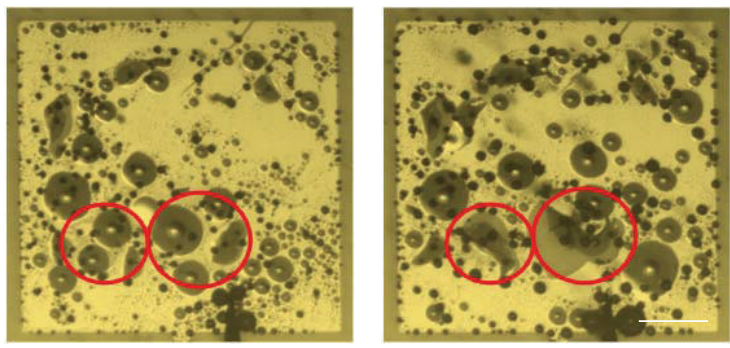

(b)
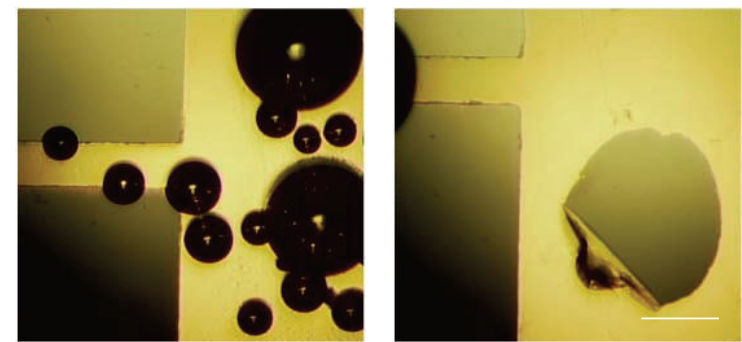

(d)

FIgure 4: Single-bubble collapse on ultrathin Au microelectrodes with a thickness of 40 to $60 \mathrm{~nm}$. The damage pits are induced by singlebubble collapse at an activation voltage of (a) $1.6 \mathrm{~V}$, (b) $1.8 \mathrm{~V}$, (c) $2.0 \mathrm{~V}$, and (d) $5.0 \mathrm{~V}$. The left and right of each image are the images before and after single-bubble collapse, respectively. Scale bars of ((a)-(c)) are $100 \mu \mathrm{m}$ and that of (d) is $50 \mu \mathrm{m}$.

calculating the pressure of tiny bubble collapse, a stand-off parameter can be calculated by $\gamma=d / r_{0}$, where $d$ is a distance between a surface of interest and a bubble center of interest and $r_{0}$ is the radius of the bubble. Figure 3(c) shows that our experimental conditions have a stand-off parameter of 1.0 at which the impulsive stress resulting from bubble collapse is directly delivered to Au microelectrodes (or micropatterns) of interest without attenuation in liquid (i.e., DI water, DPBS, etc.). Thus, our experimental samples can be most severely damaged by bubble collapse.

\subsection{Single-Bubble Collapse on Ultrathin Gold Microelectrode.} The failure mechanism caused by single-bubble collapse was examined with the experimental sample I having a $\mathrm{Cr} / \mathrm{Au}$ layer $(5 \mathrm{~nm} / 50 \pm 10 \mathrm{~nm}$ in thickness) modified with a thiolfunctionalized RGD on Pyrex glass. An applied voltage was adjusted from $1.6 \mathrm{~V}$ to $5.0 \mathrm{~V}$ to control the size of the electrolytically generated bubbles [see Figure 3(a)]. Figure 4 shows a variety of the failure shapes on the Au microelectrodes resulting from single-bubble collapse at a voltage of 1.6, $1.8,2.0$, and $5.0 \mathrm{~V}$.

The experimental results demonstrate several remarkable trends. First of all, the failures on ultrathin Au microelectrode (having a thickness of less than $100 \mathrm{~nm}$ ) caused by singlebubble collapse are observed as stress band formation in the Pyrex glass substrate from the pressure load. Thus, the failures due to single-bubble collapse are mainly generated on the underlying Pyrex substrate (refer to Figure 5(a)) instead of on the $\mathrm{Au} / \mathrm{Cr} /$ Pyrex interface (delamination) [14].

Secondly, for the ultrathin Au microelectrodes having a thickness of less than $100 \mathrm{~nm}$, the physical damages are mainly made by the collapse of a single large bubble formed through the growth or merging process of small bubbles. With an applied voltage higher than the decomposition potential of water (i.e., $1.2 \mathrm{~V}$ ), multiple small bubbles were generated on the surface of the experimental samples, followed by the growth or mild merging of the small bubbles into large ones. When the size of the grown or coalesced bubbles reached unstable bubble equilibrium radius [15], the collapse of the bubbles began and then exerted an impact load (or pressure) on the experimental samples, thus resulting in noticeable failures on the Au microelectrodes. A pressure load generated by single-bubble collapse, $P_{b}$, can be calculated by the water-hammer pressure model [16]:

$$
P_{b}=\frac{\rho_{l} \mathcal{c}_{l} \rho_{s} \mathcal{c}_{s}}{\rho_{l} \mathcal{c}_{l}+\rho_{s} c_{s}} v_{\text {imp }}
$$

where $\rho_{1}$ and $c_{l}$ are the density and sound speed of a liquid media, $\rho_{s}$ and $c_{s}$ are the density and sound speed of a solid surface, and $v_{\text {imp }}$ is the impingement velocity of a water hammer. Here, the impingement velocity can be expressed as $v_{\text {imp }}=\xi \gamma^{2} \sqrt{\Delta P / \rho_{l}}$, where $\xi$ is an experimental parameter determined by a stand-off parameter (e.g., $\xi=9.0$ at $\gamma=1.0$ ) [17] and $\Delta P$ is a pressure difference between the inside and the outside of a bubble that is correlated to the radius of the bubble, $r_{0}$, and the surface tension of a surface of interest, $\sigma$, given by $\Delta P=2 \sigma / r_{0}$. The pressure load in (1) can therefore be rewritten as

$$
P_{b}=\frac{\rho_{l} \mathcal{c}_{l} \rho_{s} \mathcal{c}_{s}}{\rho_{l} \mathcal{c}_{l}+\rho_{s} c_{s}} \xi \gamma^{2} \sqrt{\frac{\Delta P}{\rho_{l}}}=\frac{\rho_{l} \mathcal{c}_{l} \rho_{s} \mathcal{c}_{s}}{\rho_{l} \mathcal{c}_{l}+\rho_{s} c_{s}} \xi \gamma^{2} \sqrt{\frac{2 \sigma}{\left(r_{0} \rho_{l}\right)}} .
$$

Considering our experimental conditions (i.e., the generation, growth (or merging), and collapse of the tiny bubbles 

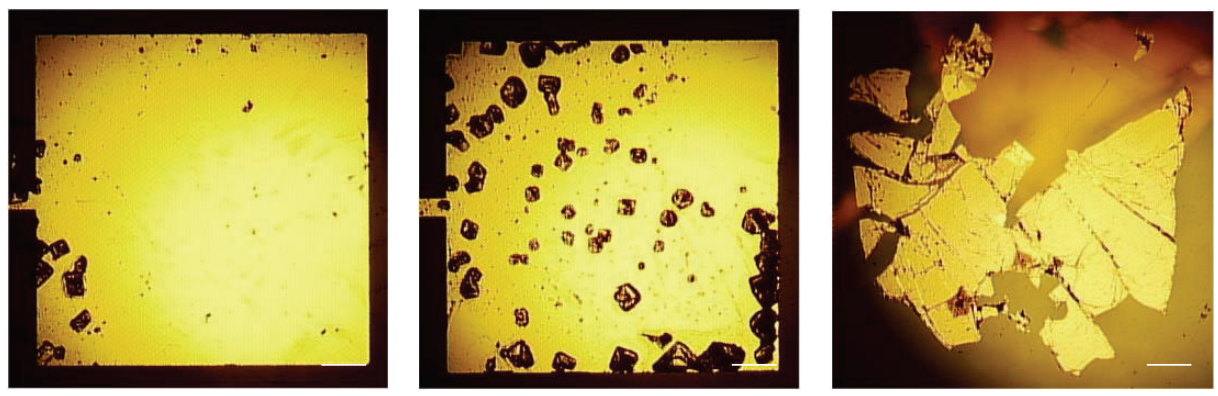

(a)
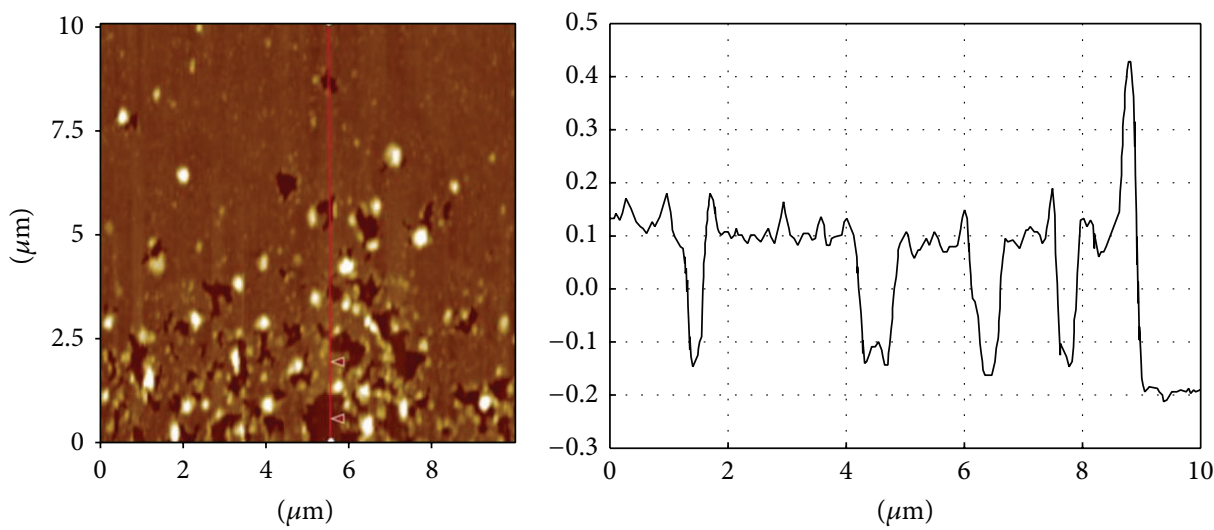

(b)

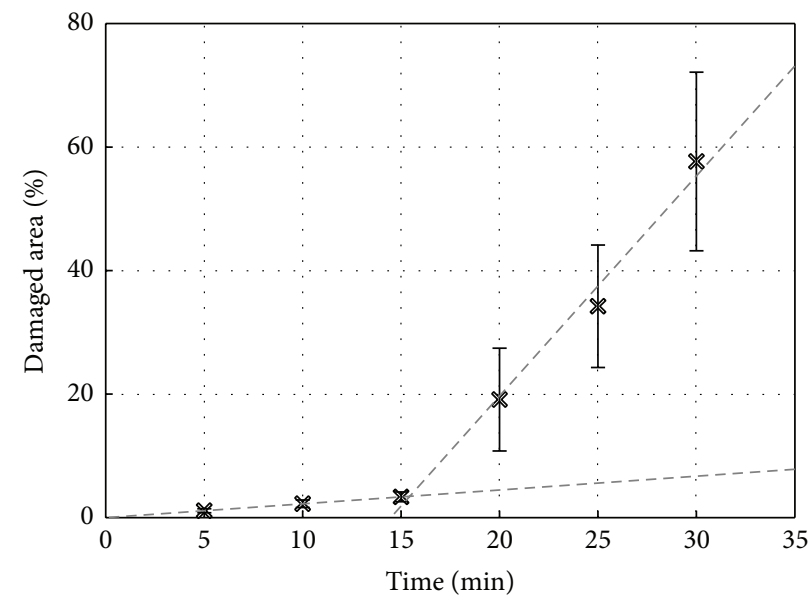

(c)

FIgURE 5: Repetitive bubble collapse on thick Au microelectrodes. (a) The damage pits on the thick Au microelectrodes at an activation voltage of $7.0 \mathrm{~V}$ obtained at 10 minutes (left), 15 minutes (middle), and 20 minutes (right) after bubble collapse. (b) The surface profile of the damage pits measured by AFM. (c) Percentage of the damaged area made by repetitive bubble collapse on 100 to $120 \mathrm{~nm}$ thick Au microelectrodes as a function of activation time at an activation voltage of $7.0 \mathrm{~V}$. The damaged area increases at 15 minutes after activation. Scale bars of (b) are $100 \mu \mathrm{m}$.

in DI water at room temperature) where $\rho_{1}=998.2 \mathrm{~kg} / \mathrm{m}^{3}$, $c_{l}=1481.0 \mathrm{~m} / \mathrm{s}, \rho_{s}=19300.0 \mathrm{~kg} / \mathrm{m}^{3}, c_{s}=2030.0 \mathrm{~m} / \mathrm{s}$, $\xi=9.0, \gamma=1.0, \sigma=72.0 \mathrm{mN} / \mathrm{m}$, and $r_{0}=50-100 \mu \mathrm{m}$, the pressure load is calculated to be $21.8\left(r_{0}=50.0 \mu \mathrm{m}\right)-$ $30.8\left(r_{0}=25.0 \mu \mathrm{m}\right) \mathrm{MPa}$. To cause physical failures on the $\mathrm{Au}$ microelectrodes, the pressure load produced by the collapse of a single large bubble after growth or merging needs to be higher than the tensile strength of a Pyrex glass wafer because the ultrathin $\mathrm{Au}$ layer on the Pyrex substrate attenuates the water-hammer pressure. This is in well agreement with the well-known tensile strength of a Pyrex glass wafer [6.9 MPa (without tempering), 20.7 MPa (with tempering)].

Thirdly, for the single large bubble that is finally formed not through the growth process of a small bubble but through the merging process of them, it is also important to note that the shape of the damaged pits caused by single-bubble 
collapse is determined by the merging process. A direction of the water-hammer pressure is normal to a direction of the merging process. For example, the damage pit has a triangular shape when a single large bubble, formed by the merging of three small bubbles, collapses [see Figure 4(a)]. In short, our experimental observation clearly indicates that the immediate failure of ultrathin $\mathrm{Au}$ microelectrodes (40 to $60 \mathrm{~nm}$ in thickness) under electrolysis is governed by the collapse of a single bubble generated through the growth or merging process of small bubbles.

3.3. Repetitive Bubble Collapse on Thick Gold Microelectrode. For thick microelectrodes having a $100-120 \mathrm{~nm}$ thick $\mathrm{Cr} / \mathrm{Au}$ layer modified with a thiol-functionalized RGD on Pyrex glass (sample II), similar experiments were performed at a relatively high voltage of $7 \mathrm{~V}$ to observe the failure mechanism of thick Au microelectrode under DI water electrolysis. This voltage led to repetitive collapse of the large bubbles made by the violent merging process of small bubbles, at which we characterize the effect of the repetitive bubble collapse on the damage pits of the sample II.

Figure 5(a) shows the microscope images of the damage pits on the thick Au microelectrodes caused by repetitive bubble collapse at $7 \mathrm{~V}$, taken 15,20 , and 30 minutes after bubble collapse. As might be expected, the size of the damage pits was in direct proportion to activation time at a given time. This is of course because an increase in the activation time results in an increase in the number of repetitive bubble collapses. Next, looking into the location of the damage pits, the bubbles (or damage pits) were concentrated in the edges of the $\mathrm{Au}$ microelectrodes rather than the middle. This phenomenon is understood by the fact that the edges have more surface density due to the height of the thick $\mathrm{Au}$ layer than the middle, thus having relatively frequent bubble formation and collapse thereon. To determine the position of the damage pits in the depth direction, their surface profile was measured with AFM for 5 thick gold samples, as shown in Figure 5(b). It was observed that the depth of the damage pits was larger than the thickness of $\mathrm{Cr} / \mathrm{Au}$ layer. This means that the failures are on the underlying Pyrex substrate instead of on the $\mathrm{Au} / \mathrm{Cr} /$ Pyrex interface, as explained above.

Figure 5(c) shows a percentage of the damaged area induced by repetitive bubble collapse on 100 to $120 \mathrm{~nm}$ thick Au microelectrodes at $7.0 \mathrm{~V}$. There was another fact that drew our attention, that is, a transition in the slope of the lines. This indicates that the failure process of thick Au microelectrodes is composed of two different stages. At the first stage (i.e., crack initiation corresponding to 0 to 15 minutes), almost no damage pits observable with an upright microscope exist but microcracks are believed to start to initiate and propagate at the underlying Pyrex substrate by repetitive bubble collapse (although the microcracks are unable to be observed due to the opacity of gold). At the second stage (i.e., damage expansion corresponding to 15 to 30 minutes), the energy accumulated by repetitive bubble collapse inflicts noticeable physical damages on the $\mathrm{Au}$ microelectrodes at 15 minutes after bubble collapse and the physical damages dramatically increase up to 30 minutes. Moreover, it was observed that, after initial physical damages were formed on the Au layer, subsequently generated damages were mainly located near the boundaries of the initial damages. This is because the boundaries have more surface density due to the height of the thick Au layer, like the location of the damage pits.

A cyclic fatigue stress caused by repetitive bubble collapse can be calculated as follows. The radius of tiny bubbles, $r$, is assumed to have a Gaussian distribution with a mean radius, $m$, of $27.2 \mu \mathrm{m}$ and a standard deviation, $s$, of $4.4 \mu \mathrm{m}$ (see Figure 3(a)) which can be described as $\Phi(r)=$ $1 /(\sqrt{2 \pi} s) \exp \left(-(r-m)^{2} / 2 s^{2}\right)$, where $\Phi$ is a Gaussian function. Together with (2), the cyclic fatigue stress, $P_{c r}$, is statistically expressed as

$$
P_{c r}=\int_{r_{\min }}^{r_{\max }} \Phi(r) \cdot P_{b}(r) d r,
$$

where $r_{\max }$ and $r_{\min }$ are the maximum and minimum radii of the tiny bubbles at $7 \mathrm{~V}$ which are set as $44.3 \mu \mathrm{m}$ and $0.1 \mu \mathrm{m}$, respectively (see Figure 3(a)). The cyclic fatigue stress is estimated as $29.7 \mathrm{MPa}$ that is higher than the tensile strength of a Pyrex glass wafer. However, single-bubble collapse fails to cause damage pits on the Au microelectrodes due to the thick $\mathrm{Cr} / \mathrm{Au}$ layer of $100-120 \mathrm{~nm}$ thickness that attenuates the water-hammer pressure induced by bubble collapse. Instead, the accumulated energy (or loading) caused by repetitive bubble collapse is responsible for physical damages on the thick Au microelectrodes. In other words, the damage mechanism of the thick Au microelectrodes (100 to $120 \mathrm{~nm}$ in thickness) is governed by a fatigue process in which there are repeated loading and unloading through multiple bubble collapse.

Our experimental results give us clear guidelines for the design and use of $\mathrm{Au}$ microelectrodes that are essentially used in bioelectronics applications. An electrical input is recommended to be lower than the decomposition potential of water to prevent the Au microelectrodes from failing and inhibit cells or tissues from being damaged. When the applied voltage needs to be higher than the decomposition potential, we need to increase the thickness of the Au microelectrodes (e.g., more than $200 \mathrm{~nm}$ ) or limit the total operating time of the Au microelectrodes (e.g., less than 10 minutes). The last and ultimate guideline is, if possible, to use substrate having high mechanical strength and good adhesion to $\mathrm{Cr} / \mathrm{Au}$.

\section{Conclusions}

In summary, two failure mechanisms of Au microelectrodes that are commonly used in bioelectronics applications have been characterized by generating and collapsing tiny hydrogen bubbles in proximity of the surface of the Au microelectrodes through deionized water electrolysis. For ultrathin $\mathrm{Au}$ microelectrodes having a thickness of 40 to $60 \mathrm{~nm}$, the water-hammer pressure induced by the collapse of a single large bubble (generated through the growth or merging process of small bubbles) causes physical failures on the underlying Pyrex substrate, thus making permanent damage pits. On the other hand, for thick Au microelectrodes having a thickness of 100 to $120 \mathrm{~nm}$, the accumulated energy caused by repeated loading and unloading through multiple bubble 
collapse results in physical damages through fatigue process. Extrapolation of this study to other microelectrodes used in bioelectronics applications might help us to investigate the physics behind bubble related phenomena such as cavitation erosion and ultrasonic cleaning.

\section{Conflict of Interests}

The authors declare that there is no conflict of interests regarding the publication of this paper.

\section{Acknowledgments}

This research was supported by the Converging Research Center Program funded by the Ministry of Science, ICT, and Future Planning (Project no. 2014048778) and the Inha University Research Grant (Grant no. INHA-47287).

\section{References}

[1] M. S. Plesset and R. B. Chapman, "Collapse of an initially spherical vapour cavity in the neighbourhood of a solid boundary," Journal of Fluid Mechanics, vol. 47, no. 2, pp. 283-290, 1971.

[2] J. A. Rooney, "Hemolysis near an ultrasonically pulsating gas bubble," Science, vol. 169, no. 3948, pp. 869-871, 1970.

[3] J. Silberstein, C. M. Lakin, and J. K. Parsons, "Shock wave lithotripsy and renal hemorrhage," Reviews in Urology, vol. 10, no. 3, pp. 236-241, 2008.

[4] R. E. A. Arndt, "Cavitation in fluid machinery and hydraulic structures," Annual Review of Fluid Mechanics, vol. 13, no. 1, pp. 273-326, 1981.

[5] X. Escaler, E. Egusquiza, M. Farhat, F. Avellan, and M. Coussirat, "Detection of cavitation in hydraulic turbines," Mechanical Systems and Signal Processing, vol. 20, no. 4, pp. 983-1007, 2006.

[6] C. Mishra and Y. Peles, "Development of cavitation in refrigerant (R-123) flow inside rudimentary microfluidic systems," Journal of Microelectromechanical Systems, vol. 15, no. 5, pp. 1319-1329, 2006.

[7] W. Kim, T.-H. Kim, J. Choi, and H.-Y. Kim, "Mechanism of particle removal by megasonic waves," Applied Physics Letters, vol. 94, no. 8, Article ID 081908, 2009.

[8] C. F. Naude and A. T. Ellis, "On the mechanism of cavitation damage by nonhemispherical cavities collapsing in contact with a solid boundary," Journal of Basic Engineering, vol. 83, no. 4, pp. 648-656, 1961.

[9] M. S. Plesset and A. Prosperetti, "Bubble dynamics and cavitation," Annual Review of Fluid Mechanics, vol. 9, no. 1, pp. 145$185,1977$.

[10] G. Karp, Cell and Molecular Biology: Concepts and Experiments, John Wiley \& Sons, New York, NY, USA, 2009.

[11] S.-H. Yoon, J. Chang, L. Lin, and M. R. K. Mofrad, "A biological breadboard platform for cell adhesion and detachment studies," Lab on a Chip, vol. 11, no. 20, pp. 3555-3562, 2011.

[12] S.-H. Yoon and M. R. K. Mofrad, "Cell adhesion and detachment on gold surfaces modified with a thiol-functionalized RGD peptide," Biomaterials, vol. 32, no. 30, pp. 7286-7296, 2011.

[13] S. Lee, W. Sutomo, C. Liu, and E. Loth, "Micro-fabricated electrolytic micro-bubblers," International Journal of Multiphase Flow, vol. 31, no. 6, pp. 706-722, 2005.
[14] X. Yin and K. Komvopoulos, "Dynamic finite element analysis of failure in alternating phase-shift masks caused by megasonic cleaning," IEEE Transactions on Components and Packaging Technologies, vol. 33, no. 1, pp. 46-55, 2010.

[15] C. E. Brennen, Cavitation and Bubble Dynamics, Cambridge University Press, Oxford, UK, 1995.

[16] S. S. Cook, "Erosion by Water-Hammer," Proceedings of the Royal Society of London A, vol. 119, no. 783, pp. 481-488, 1928.

[17] J. R. Blake and D. C. Gibson, "Cavitation bubbles near boundaries," Annual Review of Fluid Mechanics, vol. 19, pp. 99-123, 1987. 

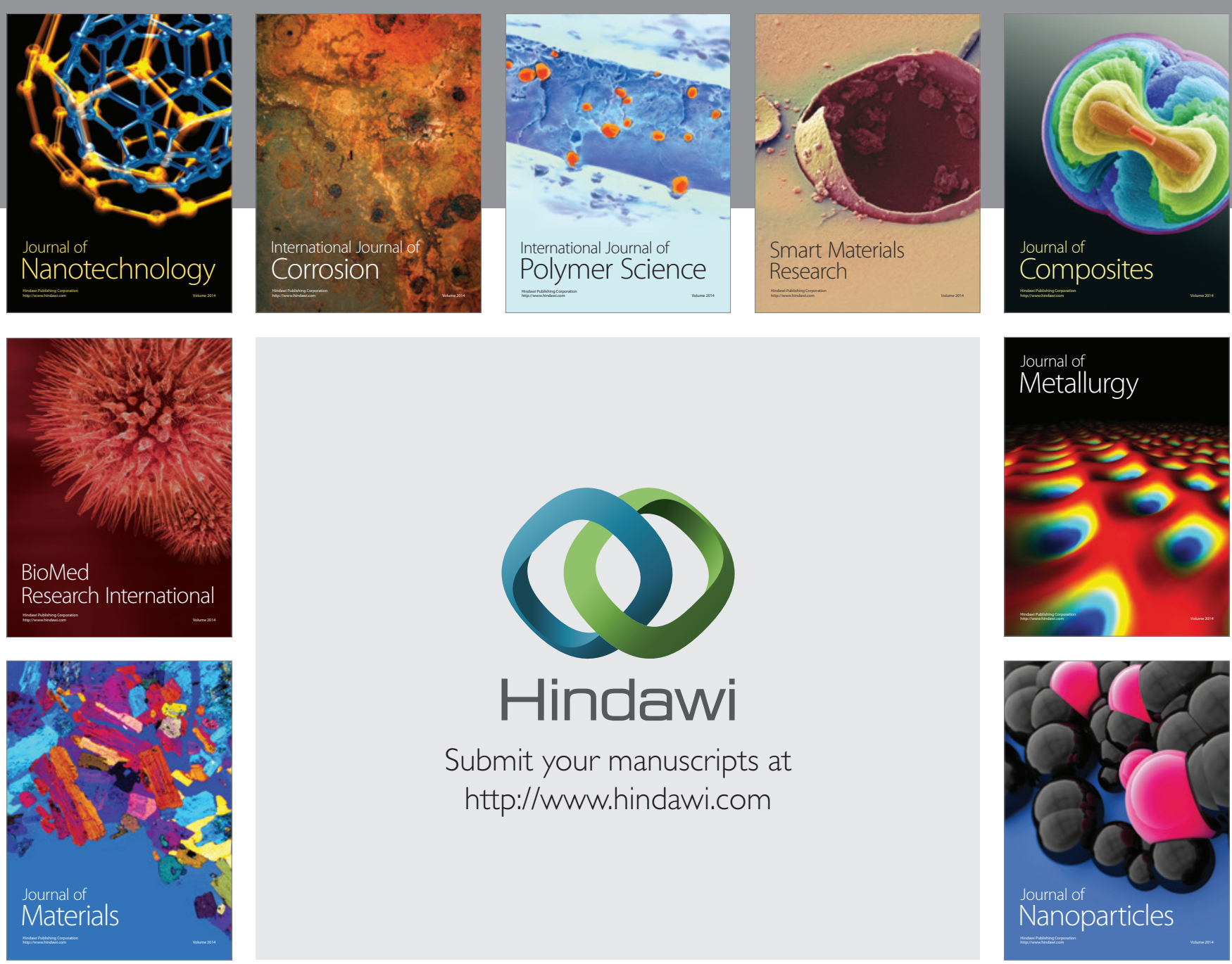

Submit your manuscripts at http://www.hindawi.com
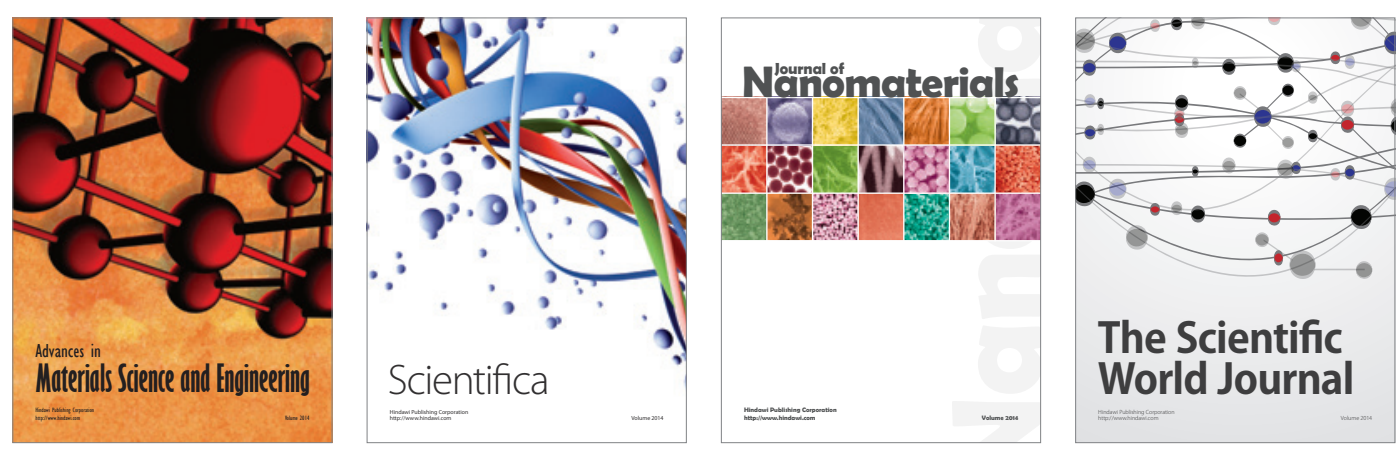

\section{The Scientific World Journal}
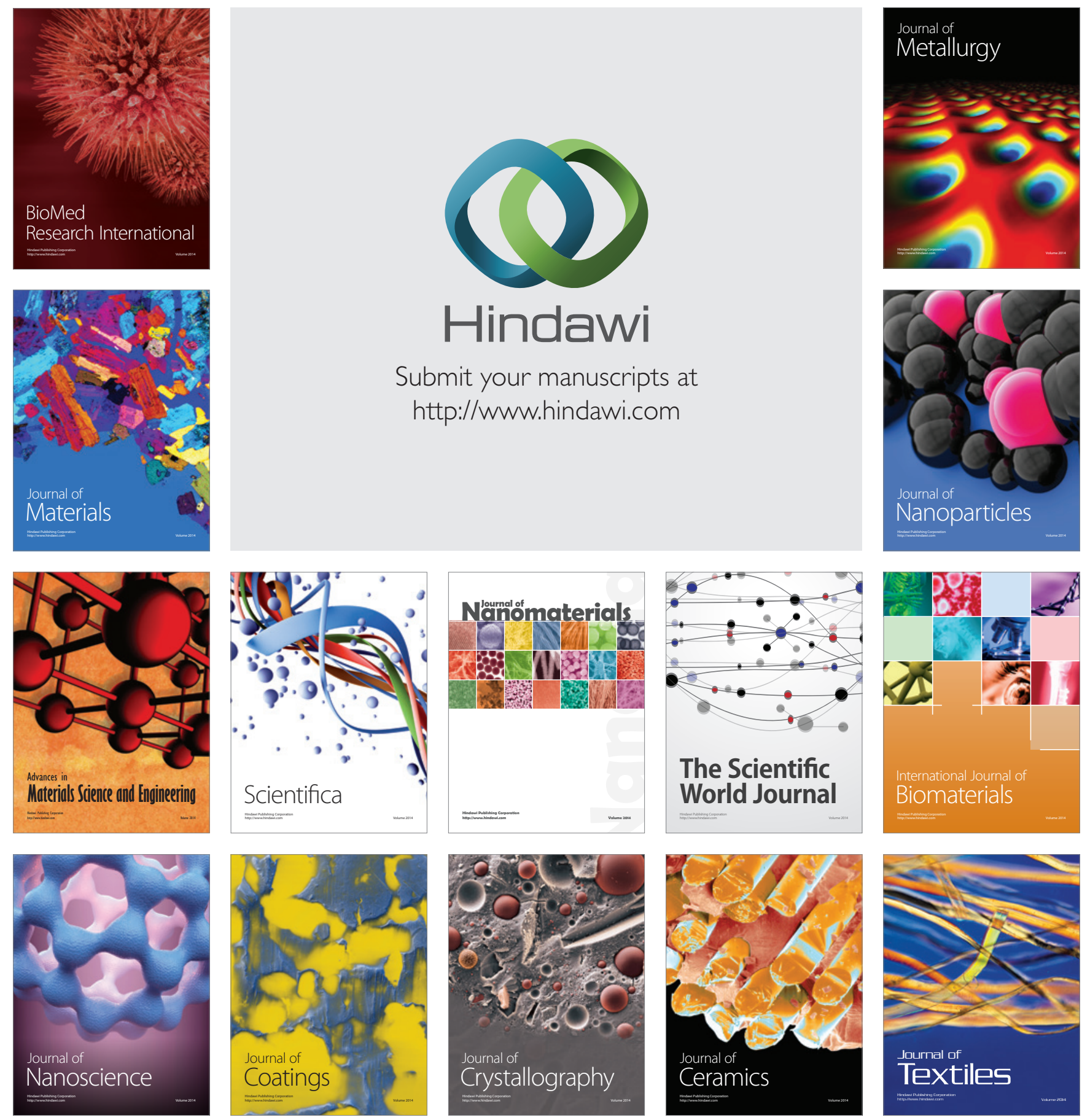